\title{
Discontinuous Galerkin Methods for Second-Order Elliptic PDE with Low-Regularity Solutions
}

\author{
Thomas P. Wihler • Béatrice Rivière
}

Received: 11 February 2010 / Revised: 25 May 2010 / Accepted: 27 May 2010 /

Published online: 17 June 2010

(C) Springer Science+Business Media, LLC 2010

\begin{abstract}
In this paper we derive an a priori error analysis for interior penalty discontinuous Galerkin finite element discretizations of the Poisson equation with exact solution in $W^{2, p}$, $p \in(1,2]$. We show that the DGFEM converges at an optimal algebraic rate with respect to the number of degrees of freedom.
\end{abstract}

Keywords Elliptic PDE · Discontinuous Galerkin methods · Low regularity solutions

\section{Introduction}

In a bounded open Lipschitz domain $\Omega \subset \mathbb{R}^{2}$, we consider the elliptic model problem

$$
\begin{aligned}
-\Delta u=f & \text { in } \Omega, \\
u=0 & \text { on } \partial \Omega,
\end{aligned}
$$

where $f \in L^{p}(\Omega)$, for some $p \in(1, \infty]$, is a given function.

In this paper, we use the following standard notation: For an open set $D \subset \mathbb{R}^{n}$ and $p \in$ $[1, \infty]$, we denote by $L^{p}(D)$ the space of all (scalar-valued) functions $u$ on $D$ for which the corresponding $L^{p}$-norm,

$$
\|u\|_{L^{p}(D)}= \begin{cases}\left(\int_{D}|u(\boldsymbol{x})|^{p} \mathrm{~d} \boldsymbol{x}\right)^{\frac{1}{p}} & \text { for } p \in[1, \infty), \\ \operatorname{ess} \sup _{\boldsymbol{x} \in D}|u(\boldsymbol{x})| & \text { for } p=\infty,\end{cases}
$$

B. Rivière research was partially supported by NSF grant DMS 0810422.

T.P. Wihler $(\bowtie)$

Mathematics Institute, University of Bern, Sidlerstrasse 5, 3012 Bern, Switzerland

e-mail: wihler@math.unibe.ch

B. Rivière

Computational and Applied Mathematics Department, Rice University, 6100 Main Street, MS-134,

Houston, TX 77005-1892, USA

e-mail: riviere@caam.rice.edu 
is bounded. Furthermore, for $s \in \mathbb{N}, W^{s, p}(D)$ signifies the standard Sobolev space of all functions whose (weak) derivatives up to order $s$ are bounded in the $L^{p}$-norm. The corresponding norms and semi-norms are denoted by $\|\cdot\|_{W^{s, p}(D)}$ and $|\cdot|_{W^{s, p}(D)}$, respectively. Moreover, $W_{0}^{s, p}(D)$ is the subspace of functions in $W^{s, p}(D)$ with zero trace on $\partial D$. For vector-valued functions, the $L^{p}$-norms are defined like in (3) (the absolute values are replaced by the Euclidean norm on $\mathbb{R}^{n}$ ) and, for simplicity, are denoted like the corresponding norms for scalar-valued functions. Finally, for $p \in[1, \infty]$, we denote by $p^{*} \in[1, \infty]$ the real number for which there holds $\frac{1}{p}+\frac{1}{p^{*}}=1\left(p^{*}=1\right.$ if $p=\infty$, and $p^{*}=\infty$ if $\left.p=1\right)$.

The standard weak formulation of (1)-(2) is to find $u \in W_{0}^{1,2}(\Omega)$ such that

$$
a(u, v)=\ell(v) \quad \forall v \in W_{0}^{1,2}(\Omega),
$$

where

$$
a(u, v)=\int_{\Omega} \nabla u \cdot \nabla v \mathrm{~d} \boldsymbol{x}, \quad \ell(v)=\int_{\Omega} f v \mathrm{~d} \boldsymbol{x} .
$$

Given that the solution of (1)-(2) belongs to $W_{0}^{1,2}(\Omega)$, it is well-known that the standard conforming finite element method converges; see, e.g., [7, Theorem 3.2.3]. This follows from the fact that, firstly, the linear forms (5) and their corresponding standard FEM forms for (1)-(2) are formally the same (where the FEM is defined on a subspace of the solution space $W_{0}^{1,2}(\Omega)$ ) and, secondly, from the density of the conforming finite element space in $W_{0}^{1,2}(\Omega)$. Indeed, convergence is still available even if the solution of (1)-(2) is exactly in $W_{0}^{1,2}(\Omega)$. However, in that case, even though the finite element method converges, there is typically no algebraic convergence rate with respect to the number of degrees of freedom (i.e., the dimension of the corresponding finite element space). An analogous result for discontinuous Galerkin methods has recently been proved in [10].

There is a variety of articles which deal with DG discretizations of elliptic problems under standard regularity assumptions (e.g., for solutions in $W^{2,2}$ or $W^{\frac{3}{2}+\epsilon, 2}$ with $\epsilon>0$ ); we refer to, e.g., $[2-4,6,9,12,14,15,18]$ and the references therein for different types of DG methods. Obtaining a priori error estimates and, particularly, algebraic convergence rates for DG methods for low regularity solutions may be accompanied by some potential difficulties. For instance, the numerical fluxes along element interfaces appearing in DG schemes need to be carefully dealt with. Particularly, note that for functions in $W_{0}^{1,2}(\Omega)$ the trace of the normal gradient on element boundaries does not necessarily belong to $L^{2}$ and needs to be controlled in alternative norms. For elliptic problems with corner singularities in polygons, with solutions belonging to some weighted Sobolev spaces (based on a weighted $W^{2,2}$ norm), DG methods have been analyzed in [16, 19-21], for example.

In this paper, we will investigate interior penalty DG discretizations of (1)-(2) with solutions in $W^{2, p}(\Omega)$ with $p \in(1,2]$. We develop a priori error estimates in the standard energy norm and prove that the DG still converges at an (optimal) algebraic rate even if $p<2$.

The article is organized as follows: In Sect. 2 we briefly review a Sobolev embedding result, discuss the variational formulation (4) of (1)-(2), and recall the regularity of the problem. Furthermore, Sect. 3 presents the interior penalty DG schemes. In Sect. 4, we consider some interpolation estimates, and Sect. 5 gives the main result. Finally, we include some numerical results in Sect. 6 and complete the article with a few concluding remarks in Sect. 7. 


\section{Regularity}

The following Sobolev embedding result will be essential in our analysis. It is an excerpt from [1, Theorem 4.12].

Lemma 2.1 Suppose that $p \in[1, \infty)$. Furthermore, let $D \subset \mathbb{R}^{2}$ be a bounded open Lipschitz domain. Then, the embedding $W^{1, p}(D) \hookrightarrow L^{q}(D)$ is continuous for all $q \in[1, \infty)$ if $p \geq 2$, and for all $q \in\left[1, \frac{2 p}{2-p}\right]$ if $p<2$.

Lemma 2.2 For any $f \in L^{p}(\Omega)$, with $p \in(1, \infty]$ the variational formulation (4) has a unique solution in $W_{0}^{1,2}(\Omega)$.

Proof The coercivity and continuity of the bilinear $a$ with respect to the $W^{1,2}(\Omega)$-norm follow from standard arguments. In order to prove the continuity of $l$, we first use Hölder's inequality,

$$
|\ell(v)| \leq\|f\|_{L^{p}(\Omega)}\|v\|_{L^{p^{*}}(\Omega)} .
$$

Using Lemma 2.1, there holds

$$
|\ell(v)| \leq C\|f\|_{L^{p}}\|v\|_{W^{1,2}(\Omega)} .
$$

Hence, $\ell$ is continuous, and the proof is complete.

Furthermore, the ensuing regularity result for (1)-(2) is available; cf. [8].

Proposition 2.3 There exists a constant $\mu>1$ such that for any $f \in L^{p}(\Omega)$ with $p \in(1, \mu)$ the solution of (1)-(2) belongs to $W^{2, p}(\Omega)$. Furthermore, there holds the elliptic regularity estimate

$$
\|u\|_{W^{2, p}(\Omega)} \leq C\|f\|_{L^{p}(\Omega)},
$$

where $C>0$ is a constant independent of $u$.

\section{Discontinuous Galerkin Discretization}

We will now introduce the (interior penalty) discontinuous Galerkin discretizations that will be analyzed in this paper.

\subsection{Meshes, Spaces, and Element Boundary Operators}

Let us consider shape-regular meshes $\mathcal{T}$ that partition $\Omega$ into open disjoint elements $\{K\}_{K \in \mathcal{T}}$, i.e., $\bar{\Omega}=\bigcup_{K \in \mathcal{T}} \bar{K}$. For simplicity, we do not consider hanging nodes in this work, even though the subsequent analysis could be extended easily to 1-irregular meshes, for example. Each element $K \in \mathcal{T}$ is an image of the open reference triangle $\widehat{T}=\left\{\left(\widehat{x}_{1}, \widehat{x}_{2}\right):-1<\right.$ $\left.\widehat{x}_{1}<1,-1<\widehat{x}_{2}<-\widehat{x}_{1}\right\}$ or of the open reference square $\widehat{Q}=(-1,1)^{2}$. By $h_{K}$, we denote the diameter of an element $K \in \mathcal{T}$. The elemental diameters are stored in a vector $\boldsymbol{h}$ given by $\boldsymbol{h}=\left[h_{K}\right]_{K \in \mathcal{T}}$. 
Moreover, we will define some suitable element boundary operators that are required for the DG method. To this end, we denote by $\mathcal{E}_{\mathcal{I}}$ the set of all interior edges and by $\mathcal{E}_{\mathcal{B}}$ the set of all boundary edges in $\mathcal{T}$. In addition, let $\mathcal{E}=\mathcal{E}_{\mathcal{I}} \cup \mathcal{E}_{\mathcal{B}}$. The boundary $\partial K$ of an element $K$ and the sets $\partial K \backslash \partial \Omega$ and $\partial K \cap \partial \Omega$ will be identified in a natural way with the corresponding subsets of $\mathcal{E}$.

Let $K_{\sharp}$ and $K_{b}$ be two adjacent elements of $\mathcal{T}$, and $\boldsymbol{x}$ an arbitrary point on the interior face $e \in \mathcal{E}_{\mathcal{I}}$ given by $e=\partial K_{\sharp} \cap \partial K_{b}$. Furthermore, let $v$ and $\boldsymbol{q}$ be scalar- and vector-valued functions, respectively, that are sufficiently smooth inside each element $K_{\sharp / b}$. By $\left(v_{\sharp / b}, \boldsymbol{q}_{\sharp / b}\right)$, we denote the traces of $(v, \boldsymbol{q})$ on $e$ taken from within the interior of $K_{\sharp / b}$, respectively. Then, the averages of $v$ and $\boldsymbol{q}$ at $\boldsymbol{x} \in e$ are given by

$$
\langle\langle v\rangle\rangle=\frac{1}{2}\left(v_{\sharp}+v_{b}\right), \quad\langle\langle\boldsymbol{q}\rangle\rangle=\frac{1}{2}\left(\boldsymbol{q}_{\sharp}+\boldsymbol{q}_{b}\right),
$$

respectively. Similarly, the jumps of $v$ and $\boldsymbol{q}$ at $\boldsymbol{x} \in e$ are given by

$$
\llbracket v \rrbracket=v_{\sharp} \boldsymbol{n}_{K_{\sharp}}+v_{b} \boldsymbol{n}_{K_{b}}, \quad \llbracket \boldsymbol{q} \rrbracket=\boldsymbol{q}_{\sharp} \cdot \boldsymbol{n}_{K_{\sharp}}+\boldsymbol{q}_{b} \cdot \boldsymbol{n}_{K_{b}},
$$

respectively. Here, for $K \in \mathcal{T}$, we denote by $\boldsymbol{n}_{K}$ the unit outward normal vector of $\partial K$, respectively. On a boundary face $e \in \mathcal{E}_{\mathcal{B}}$, we set $\langle\langle v\rangle\rangle=v,\langle\langle\boldsymbol{q}\rangle\rangle=\boldsymbol{q}$ and $\llbracket v \rrbracket=v \boldsymbol{n}$, with $\boldsymbol{n}$ denoting the unit outward normal vector on the boundary $\partial \Omega$.

For a given finite element mesh $\mathcal{T}$ and a polynomial degree $l \geq 1$, consider the DG space

$$
V_{\mathrm{DG}}(\mathcal{T})=\left\{v \in L^{2}(\Omega):\left.v\right|_{K} \in \mathcal{S}_{l}(K) \forall K \in \mathcal{T}\right\},
$$

where, for $K \in \mathcal{T}, \mathcal{S}_{l}(K)$ signifies either the space $\mathcal{P}_{l}(K)$ of all polynomials of total degree at most $l$ on $K$, or the space $\mathcal{Q}_{l}(K)$ of all polynomials of degree at most $l$ in each coordinate direction. Furthermore, for $p \in[1, \infty]$ and $s \in \mathbb{N}$, let

$$
W^{s, p}(\Omega, \mathcal{T})=\left\{v \in L^{p}(\Omega):\left.v\right|_{K} \in W^{s, p}(K) \forall K \in \mathcal{T}\right\}
$$

We will discretize (1)-(2) by an interior penalty discontinuous Galerkin method (see, e.g., $[2,3,9,15,18])$ and the reference therein. More precisely, for fixed parameters $\gamma>0$, $\theta \in \mathbb{R}$, we define a DG approximation $u_{\mathrm{DG}} \in V_{\mathrm{DG}}(\mathcal{T})$ by

$$
a_{\mathrm{DG}}\left(u_{\mathrm{DG}}, v\right)=\ell_{\mathrm{DG}}(v) \quad \forall v \in V_{\mathrm{DG}}(\mathcal{T}),
$$

where

$$
\begin{aligned}
a_{\mathrm{DG}}(w, v)= & \int_{\Omega} \nabla_{h} w \cdot \nabla_{h} v \mathrm{~d} \boldsymbol{x}-\int_{\mathcal{E}}\left\langle\left\langle\nabla_{h} w\right\rangle\right\rangle \cdot \llbracket v \rrbracket \mathrm{d} s \\
& -\theta \int_{\mathcal{E}} \llbracket w \rrbracket \cdot\left\langle\left\langle\nabla_{h} v\right\rangle\right\rangle \mathrm{d} s+\gamma \int_{\mathcal{E}} h^{-1} \llbracket w \rrbracket \cdot \llbracket v \rrbracket \mathrm{d} s,
\end{aligned}
$$

and

$$
\ell_{\mathrm{DG}}(v)=\int_{\Omega} f v \mathrm{~d} \boldsymbol{x} .
$$

Here, $\nabla_{h}$ is the elementwise gradient, and $h \in L^{\infty}(\mathcal{E})$ is given by

$$
h(\boldsymbol{x})= \begin{cases}\min \left(h_{K_{\sharp}}, h_{K_{b}}\right) & \text { for } \boldsymbol{x} \in \partial K_{\sharp} \cap \partial K_{b} \in \mathcal{E}_{\mathcal{I}}, \\ h_{K} & \text { for } \boldsymbol{x} \in \partial K \cap \partial \Omega \in \mathcal{E}_{\mathcal{B}} .\end{cases}
$$


Moreover, we define the DG norm

$$
\|\| \cdot \|_{\text {DG }}=\left(\int_{\Omega}\left|\nabla_{h}(\cdot)\right|^{2} \mathrm{~d} \boldsymbol{x}+\gamma \int_{\mathcal{E}} \mathrm{h}^{-1} \mid\left[\left.[\cdot]\right|^{2} \mathrm{~d} s\right)^{\frac{1}{2}} .\right.
$$

For sufficiently large $\gamma$, the form $a_{\mathrm{DG}}$ is coercive with respect to $\|\cdot\| \|_{\mathrm{DG}}$, and hence, the $\operatorname{DGFEM}(7)$ has a unique solution $u_{\mathrm{DG}} \in V_{\mathrm{DG}}(\mathcal{T})$; cf. [15].

We will require the following estimate.

Lemma 3.1 Let $q \in[2, \infty)$. Then there exists a constant $C>0$ independent of $\boldsymbol{h}$ such that

$$
\left(\int_{\mathcal{E}} \mathrm{h}^{-1}|\llbracket v \rrbracket|^{q} \mathrm{~d} s\right)^{\frac{1}{q}} \leq C\|\| v \|_{\mathrm{DG}}
$$

for any $v \in V_{\mathrm{DG}}(\mathcal{T})$.

Proof There holds

$$
\int_{\mathcal{E}} \mathrm{h}^{-1}|\llbracket v \rrbracket|^{q} \mathrm{~d} s=\sum_{e \in \mathcal{E}}\left\|\mathrm{h}^{-\frac{1}{q}} \llbracket v \rrbracket\right\|_{L^{q}(e)}^{q} .
$$

Furthermore, using that $v$ belongs to a finite dimensional space, we have the norm equivalence (including appropriate scaling)

$$
\left\|\mathrm{h}^{-\frac{1}{q}} \llbracket v \rrbracket\right\|_{L^{q}(e)} \leq C\left\|\mathrm{~h}^{-\frac{1}{2}} \llbracket v \rrbracket\right\|_{L^{2}(e)} \quad \forall e \in \mathcal{E},
$$

where $C>0$ is a constant independent of $v$ and of $\boldsymbol{h}$. Hence, we conclude

$$
\int_{\mathcal{E}} \mathrm{h}^{-1}|\llbracket v \rrbracket|^{q} \mathrm{~d} s \leq C \sum_{e \in \mathcal{E}}\left(\int_{e} \mathrm{~h}^{-1}|\llbracket v \rrbracket|^{2} \mathrm{~d} s\right)^{\frac{q}{2}} .
$$

Now, we recall a special case of Jensen's inequality (see, e.g., [11]): Let $0<r \leq s$ and $a_{j} \geq 0, j=1,2, \ldots, n$ be real numbers, and $n \in \mathbb{N}$. Then,

$$
\left(\sum_{j=1}^{n} a_{j}^{s}\right)^{\frac{1}{s}} \leq\left(\sum_{j=1}^{n} a_{j}^{r}\right)^{\frac{1}{r}}
$$

With $r=1$ and $s=\frac{q}{2} \geq 1$, it holds that

$$
\left(\sum_{e \in \mathcal{E}}\left(\int_{e} \mathrm{~h}^{-1}|\llbracket v \rrbracket|^{2} \mathrm{~d} s\right)^{\frac{q}{2}}\right)^{\frac{2}{q}} \leq \sum_{e \in \mathcal{E}} \int_{e} \mathrm{~h}^{-1}|\llbracket v \rrbracket|^{2} \mathrm{~d} s .
$$

Inserting this inequality into (10) leads to

$$
\int_{\mathcal{E}} \mathrm{h}^{-1}|\llbracket v \rrbracket|^{q} \mathrm{~d} s \leq C\left(\sum_{e \in \mathcal{E}} \int_{e} \mathrm{~h}^{-1}|\llbracket v \rrbracket|^{2} \mathrm{~d} s\right)^{\frac{q}{2}} .
$$


Finally, noticing that

$$
\sum_{e \in \mathcal{E}} \int_{e} \mathrm{~h}^{-1}|\llbracket v \rrbracket|^{2} \mathrm{~d} s \leq \gamma^{-1}\|v\|_{\mathrm{DG}}^{2},
$$

implies the desired bound.

\subsection{Galerkin Orthogonality}

The aim of this section is the proof of a Galerkin orthogonality result. For this purpose, let us first consider an auxiliary lemma.

Lemma 3.2 Let $p \in(1,2]$, and consider two neighboring elements $K_{\sharp}, K_{b} \in \mathcal{T}$ which share an interface $e \in \mathcal{E}_{\mathcal{I}}$. Moreover, let $\Omega_{e}=\left(\bar{K}_{\sharp} \cup \bar{K}_{b}\right)^{\circ}$. Then, for $w \in W^{1, p}\left(\Omega_{e}\right)$, there holds $\left.\llbracket w \rrbracket\right|_{e}=\mathbf{0}$. Furthermore, if $w \in W^{2, p}\left(\Omega_{e}\right)$, then $\left.\llbracket \nabla w \rrbracket\right|_{e}=0$.

Proof We show the scalar case $w \in W^{1, p}\left(\Omega_{e}\right)$ only. Referring to, e.g., [13, Proposition 3.2.1], it follows that there holds

$$
\int_{e}\left(\llbracket w \rrbracket \cdot \boldsymbol{n}_{e}\right) \phi \mathrm{d} s=0 \quad \forall \phi \in C_{0}^{\infty}(e),
$$

where $\boldsymbol{n}_{e}$ is the unit normal vector on $e$ pointing from $K_{\sharp}$ to $K_{b}$, and $C_{0}^{\infty}(e)$ is the space of all smooth functions on $e$ with compact support. Furthermore, recalling the trace theorem, it follows that $\llbracket w \rrbracket \cdot \boldsymbol{n}_{e} \in L^{p}(e)$. Consequently, for any $z \in L^{p^{*}}(e)$ and $\phi \in C_{0}^{\infty}(e)$, we obtain

$$
\left|\int_{e}\left(\llbracket w \rrbracket \cdot \boldsymbol{n}_{e}\right) z \mathrm{~d} s\right|=\left|\int_{e}\left(\llbracket w \rrbracket \cdot \boldsymbol{n}_{e}\right)(z-\phi) \mathrm{d} s\right| \leq\left\|\llbracket w \rrbracket \cdot \boldsymbol{n}_{e}\right\|_{L^{p}(e)}\|z-\phi\|_{L^{p^{*}}(e)} .
$$

Thence, by density of $C_{0}^{\infty}(e)$ in $L^{p^{*}}(e)$, it follows that

$$
\int_{e}\left(\llbracket w \rrbracket \cdot \boldsymbol{n}_{e}\right) z \mathrm{~d} s=0 \quad \forall z \in L^{p^{*}}(e) .
$$

Finally, choosing $z=\operatorname{sgn}\left(\llbracket w \rrbracket \cdot \boldsymbol{n}_{e}\right)\left|\llbracket w \rrbracket \cdot \boldsymbol{n}_{e}\right|^{p-1} \in L^{p^{*}}(e)$, implies $\left\|\llbracket w \rrbracket \cdot \boldsymbol{n}_{e}\right\|_{L^{p}(e)}=0$, and thus, the proof is complete.

Moreover, we have the following result:

Proposition 3.3 Let $p \in(1,2]$. Then, the $D G$ bilinear form $a_{\mathrm{DG}}$ from (8) is well-defined on $W^{2, p}(\Omega) \times V_{\mathrm{DG}}(\mathcal{T})$. In addition, if the exact solution $u$ of $(1)-(2)$ belongs to $W^{2, p}(\Omega)$, then there holds

$$
a_{\mathrm{DG}}(u, v)=\ell_{\mathrm{DG}}(v)
$$

for all $v \in V_{\mathrm{DG}}(\mathcal{T})$.

Proof Let $w \in W^{2, p}(\Omega), v \in V_{\mathrm{DG}}(\mathcal{T})$. Then, we split the expression $a_{\mathrm{DG}}(w, v)$ into four terms,

$$
a_{\mathrm{DG}}(w, v)=T_{1}-T_{2}-\theta T_{3}+\gamma T_{4}
$$


with

$$
\begin{aligned}
T_{1} & =\int_{\Omega} \nabla w \cdot \nabla_{h} v \mathrm{~d} \boldsymbol{x}, & T_{2} & =\int_{\mathcal{E}}\langle\langle\nabla w\rangle\rangle \cdot \llbracket v \rrbracket \mathrm{d} s, \\
T_{3} & =\int_{\mathcal{E}} \llbracket w \rrbracket \cdot\left\langle\left\langle\nabla_{h} v\right\rangle\right\rangle \mathrm{d} s, & T_{4} & =\int_{\mathcal{E}} \mathrm{h}^{-1} \llbracket w \rrbracket \cdot \llbracket v \rrbracket \mathrm{d} s .
\end{aligned}
$$

The previous Lemma 3.2 implies that $\left.\llbracket w \rrbracket\right|_{\mathcal{E}} \equiv \mathbf{0}$, and thus,

$$
T_{3}=T_{4}=0 .
$$

Furthermore, using Hölder's inequality, there holds

$$
\begin{aligned}
\left|T_{1}\right| & \leq \int_{\Omega}|\nabla w|\left|\nabla_{h} v\right| \mathrm{d} \boldsymbol{x} \leq\|\nabla w\|_{L^{1}(\Omega)}\left\|\nabla_{h} v\right\|_{L^{\infty}(\Omega)} \\
& \leq\|w\|_{W^{2,1}(\Omega)}\left\|\nabla_{h} v\right\|_{L^{\infty}(\Omega)} .
\end{aligned}
$$

In addition, we have

$$
\left|T_{2}\right| \leq\|\llbracket v \rrbracket\|_{L^{\infty}(\mathcal{E})} \sum_{e \in \mathcal{E}}\|\langle\langle\nabla w\rangle\rangle\|_{L^{1}(e)} \leq C\|\llbracket v \rrbracket\|_{L^{\infty}(\mathcal{E})} \sum_{K \in \mathcal{T}}\|\nabla w\|_{L^{1}(\partial K)} .
$$

Using the trace theorem, it follows that

$$
\sum_{K \in \mathcal{T}}\|\nabla w\|_{L^{1}(\partial K)} \leq C \sum_{K \in \mathcal{T}}\|\nabla w\|_{W^{1,1}(K)} \leq C\|w\|_{W^{2,1}(\Omega)},
$$

with a constant that possibly depends on $\boldsymbol{h}$. Hence,

$$
\left|T_{2}\right| \leq C\|\llbracket v \rrbracket\|_{L^{\infty}(\mathcal{E})}\|w\|_{W^{2,1}(\Omega)} .
$$

Consequently, $a_{\mathrm{DG}}(w, v)$ is well-defined for all $w \in W^{2, p}(\Omega), v \in V_{\mathrm{DG}}(\mathcal{T})$.

It remains to prove (12). Let $u \in W^{2, p}(\Omega)$ solve (1)-(2). Then, applying Green's formula elementwise in $T_{1}$, results in

$$
T_{1}=\sum_{K \in \mathcal{T}}\left(-\int_{K} \Delta u v \mathrm{~d} \boldsymbol{x}+\int_{\partial K}\left(\nabla u \cdot \boldsymbol{n}_{K}\right) v \mathrm{~d} s\right) .
$$

Notice that, by similar arguments as before, all of the above integrals are well-defined. Furthermore, since $u$ solves (1), there holds

$$
\sum_{K \in \mathcal{T}}-\int_{K} \Delta u v \mathrm{~d} \boldsymbol{x}=\int_{\Omega} f v \mathrm{~d} \boldsymbol{x}=\ell_{\mathrm{DG}}(v) .
$$

Moreover, a few elementary manipulations show that

$$
\sum_{K \in \mathcal{T}} \int_{\partial K}\left(\nabla u \cdot \boldsymbol{n}_{K}\right) v \mathrm{~d} s=T_{2}+\int_{\mathcal{E}_{\mathcal{I}}} \llbracket \nabla u \rrbracket\langle\langle v\rangle\rangle \mathrm{d} s .
$$

Applying Lemma 3.2, we obtain $T_{1}=T_{2}+\ell_{\mathrm{DG}}(v)$. Thus, recalling (13) and (14), we see that $a_{\mathrm{DG}}(u, v)=\ell_{\mathrm{DG}}(v)$ for all $v \in V_{\mathrm{DG}}(\mathcal{T})$. 


\section{Approximation Results}

The goal of this section is to present some interpolation bounds which we will need in the ensuing error analysis. Since we are interested in estimating the error in the standard DG norm (9) (which is based on $L^{2}$-norms) in terms of appropriate $L^{p}$-norms of the exact solution, we will consider approximation results in the respective norms.

There holds:

Proposition 4.1 Let $p \in(1,2], u \in W^{l+1, p}(\Omega, \mathcal{T})$, and $l \geq 1$ be the polynomial degree in the $D G$ space $V_{\mathrm{DG}}(\mathcal{T})$. Then, there exists an interpolant $\Pi: W^{l+1, p}(\Omega, \mathcal{T}) \rightarrow V_{\mathrm{DG}}(\mathcal{T})$ such that for all $K \in \mathcal{T}$ there holds

$$
|u-\Pi u|_{W^{m, p}(K)} \leq C h_{K}^{l+1-m}\|u\|_{W^{l+1, p(K)}}, \quad 0 \leq m \leq l+1 .
$$

Furthermore, we have

$$
\begin{aligned}
& \sum_{K \in \mathcal{T}}\left(h_{K}^{-2}\|u-\Pi u\|_{L^{2}(K)}^{2}+\|\nabla(u-\Pi u)\|_{L^{2}(K)}^{2}\right) \\
& \quad \leq C\left(\sum_{K \in \mathcal{T}} h_{K}^{p(l+1)-2}\|u\|_{W^{l+1, p}(K)}^{p}\right)^{\frac{2}{p}} .
\end{aligned}
$$

In the above estimate, $C>0$ is a constant independent of $\boldsymbol{h}$.

Proof The first estimate (15) can be found in [5, 17], for example. For the proof of (16), we first apply the Sobolev embedding $W^{1, p}(K) \hookrightarrow L^{2}(K)$ for all $K \in \mathcal{T}$; cf. Lemma 2.1. Additionally, employing a scaling argument, this results in

$$
\begin{aligned}
& \sum_{K \in \mathcal{T}}\left(h_{K}^{-2}\|u-\Pi u\|_{L^{2}(K)}^{2}+\|\nabla(u-\Pi u)\|_{L^{2}(K)}^{2}\right) \\
& \leq C \sum_{m=0}^{2} \sum_{K \in \mathcal{T}}\left(h_{K}^{2 m-\frac{4}{p}}|u-\Pi u|_{W^{m, p}(K)}^{2}\right) .
\end{aligned}
$$

Recalling (15), leads to

$$
\sum_{K \in \mathcal{T}}\left(h_{K}^{-2}\|u-\Pi u\|_{L^{2}(K)}^{2}+\|\nabla(u-\Pi u)\|_{L^{2}(K)}^{2}\right) \leq C \sum_{K \in \mathcal{T}} h_{K}^{2 l+2-\frac{4}{p}}\|u\|_{W^{l+1, p}(K)}^{2} .
$$

Furthermore, applying (11) with $r=p, s=2$, we obtain

$$
\begin{aligned}
& \sum_{K \in \mathcal{T}}\left(h_{K}^{-2}\|u-\Pi u\|_{L^{2}(K)}^{2}+\|\nabla(u-\Pi u)\|_{L^{2}(K)}^{2}\right) \\
& \quad \leq C \sum_{K \in \mathcal{T}}\left(h_{K}^{l+1-\frac{2}{p}}\|u\|_{W^{l+1, p}(K)}\right)^{2} \\
& \quad \leq C\left(\sum_{K \in \mathcal{T}} h_{K}^{p(l+1)-2}\|u\|_{W^{l+1, p}(K)}^{p}\right)^{\frac{2}{p}} .
\end{aligned}
$$

This completes the proof. 


\section{Error Analysis}

In this section we will study the convergence of the DGFEM (7) for exact solutions which belong to $W^{2, p}(\Omega, \mathcal{T})$. To this end, we first split the error $e_{\mathrm{DG}}=u-u_{\mathrm{DG}}$ between the exact solution and the DG solution into two parts,

$$
e_{\mathrm{DG}}=\eta+\xi=(u-\mathrm{P} u)+\left(\mathrm{P} u-u_{\mathrm{DG}}\right),
$$

where $\mathrm{P} u \in V_{\mathrm{DG}}(\mathcal{T})$ is an arbitrary interpolant of the exact solution $u$.

Then there holds the following.

Proposition 5.1 Let $p \in(1,2]$, and $\eta$ from (17). Then,

$$
\begin{aligned}
& \left|a_{\mathrm{DG}}(\eta, v)\right| \\
& \quad \leq C\left(\|\eta\|_{\mathrm{DG}}+\left(\sum_{K \in \mathcal{T}} h_{K}^{p-2}\left(\|\nabla \eta\|_{L^{p}(K)}+h_{K}\left\|\mathrm{D}^{2} \eta\right\|_{L^{p}(K)}\right)^{p}\right)^{\frac{1}{p}}\right)\|v\|_{\mathrm{DG}}
\end{aligned}
$$

for all $v \in V_{\mathrm{DG}}(\mathcal{T})$, where $C>0$ is a constant independent of $\eta, v$, and $\boldsymbol{h}$.

Proof We split $a_{\mathrm{DG}}(\eta, v)$ into four terms,

$$
a_{\mathrm{DG}}(\eta, v)=T_{1}-T_{2}-\theta T_{3}+\gamma T_{4},
$$

with

$$
\begin{aligned}
T_{1} & =\int_{\Omega} \nabla_{h} \eta \cdot \nabla_{h} v \mathrm{~d} \boldsymbol{x}, & T_{2} & =\int_{\mathcal{E}}\left\langle\left\langle\nabla_{h} \eta\right\rangle\right\rangle \cdot \llbracket v \rrbracket \mathrm{d} s, \\
T_{3} & =\int_{\mathcal{E}} \llbracket \eta \rrbracket \cdot\left\langle\left\langle\nabla_{h} v\right\rangle\right\rangle \mathrm{d} s, & T_{4} & =\int_{\mathcal{E}} \mathrm{h}^{-1} \llbracket \eta \rrbracket \cdot \llbracket v \rrbracket \mathrm{d} s .
\end{aligned}
$$

We will estimate each of the above terms separately. For $T_{1}$, there holds

$$
\begin{aligned}
\left|T_{1}\right| & \leq \sum_{K \in \mathcal{T}} \int_{K}|\nabla \eta||\nabla v| \mathrm{d} \boldsymbol{x} \\
& \leq\left(\sum_{K \in \mathcal{T}}\|\nabla \eta\|_{L^{2}(K)}^{2}\right)^{\frac{1}{2}}\left(\sum_{K \in \mathcal{T}}\|\nabla v\|_{L^{2}(K)}^{2}\right)^{\frac{1}{2}} \\
& \leq\|\eta\|_{\mathrm{DG}}\|v\|_{\mathrm{DG}} .
\end{aligned}
$$

Furthermore, we have

$$
\left|T_{2}\right| \leq\left(\int_{\mathcal{E}}\left(\mathrm{h}^{\frac{1}{p^{*}}}\left|\left\langle\left\langle\nabla_{h} \eta\right\rangle\right\rangle\right|\right)^{p} \mathrm{~d} s\right)^{\frac{1}{p}}\left(\int_{\mathcal{E}}\left(\mathrm{h}^{-\frac{1}{p^{*}}}|\llbracket v \rrbracket|\right)^{p^{*}} \mathrm{~d} s\right)^{\frac{1}{p^{*}}} .
$$

Here, we notice that

$$
\int_{\mathcal{E}}\left(\mathrm{h}^{\frac{1}{p^{*}}}\left|\left\langle\left\langle\nabla_{h} \eta\right\rangle\right\rangle\right|\right)^{p} \mathrm{~d} s=\sum_{e \in \mathcal{E}} \int_{e}\left(\mathrm{~h}^{\frac{1}{p^{*}}}\left|\left\langle\left\langle\nabla_{h} \eta\right\rangle\right\rangle\right|\right)^{p} \mathrm{~d} s
$$




$$
\begin{aligned}
& \leq C \sum_{K \in \mathcal{T}} \int_{\partial K}\left(\mathrm{~h}^{\frac{1}{p^{*}}}\left|\left\langle\left\langle\nabla_{h} \eta\right\rangle\right\rangle\right|\right)^{p} \mathrm{~d} s \\
& \leq C \sum_{K \in \mathcal{T}} \int_{\partial K} \mathrm{~h}^{p-1}\left|\left\langle\left\langle\nabla_{h} \eta\right\rangle\right\rangle\right|^{p} \mathrm{~d} s .
\end{aligned}
$$

Due to the shape regularity of $\mathcal{T}$ it follows that

$$
\begin{aligned}
\int_{\mathcal{E}}\left(\mathrm{h}^{\frac{1}{p^{*}}}\left|\left\langle\left\langle\nabla_{h} \eta\right\rangle\right\rangle\right|\right)^{p} \mathrm{~d} s & \leq C \sum_{K \in \mathcal{T}} h_{K}^{p-1} \int_{\partial K}\left|\left\langle\left\langle\nabla_{h} \eta\right\rangle\right\rangle\right|^{p} \mathrm{~d} s \\
& \leq C \sum_{K \in \mathcal{T}} h_{K}^{p-1} \int_{\partial K}|\nabla \eta|^{p} \mathrm{~d} s .
\end{aligned}
$$

Hence,

$$
\left|T_{2}\right| \leq C\left(\sum_{K \in \mathcal{T}} h_{K}^{p-1} \int_{\partial K}|\nabla \eta|^{p} \mathrm{~d} s\right)^{\frac{1}{p}}\left(\int_{\mathcal{E}} \mathrm{h}^{-1}|\llbracket v \rrbracket|^{p^{*}} \mathrm{~d} s\right)^{\frac{1}{p^{*}}} .
$$

Noting that $p^{*} \in[2, \infty)$ we can recall Lemma 3.1. This leads to

$$
\left|T_{2}\right| \leq C\left(\sum_{K \in \mathcal{T}} h_{K}^{p-1}\|\nabla \eta\|_{L^{p}(\partial K)}^{p}\right)^{\frac{1}{p}}\|v\|_{\mathrm{DG}} .
$$

Applying the trace inequality and scaling, results in

$$
\sum_{K \in \mathcal{T}} h_{K}^{p-1}\|\nabla \eta\|_{L^{p}(\partial K)}^{p} \leq C \sum_{K \in \mathcal{T}} h_{K}^{p-1}\left(h_{K}^{-\frac{1}{p}}\|\nabla \eta\|_{L^{p}(K)}+h_{K}^{1-\frac{1}{p}}\left\|\mathrm{D}^{2} \eta\right\|_{L^{p}(K)}\right)^{p} .
$$

Therefore,

$$
\left|T_{2}\right| \leq C\left(\sum_{K \in \mathcal{T}} h_{K}^{p-2}\left(\|\nabla \eta\|_{L^{p}(K)}+h_{K}\left\|\mathrm{D}^{2} \eta\right\|_{L^{p}(K)}\right)^{p}\right)^{\frac{1}{p}}\|v\|_{\mathrm{DG}} .
$$

In addition, we have

$$
\begin{aligned}
\left|T_{3}\right| & \leq\left(\sum_{e \in \mathcal{E}}\left\|\mathrm{h}^{\frac{1}{2}}\left\langle\left\langle\nabla_{h} v\right\rangle\right\rangle\right\|_{L^{2}(e)}^{2}\right)^{\frac{1}{2}}\left(\int_{\mathcal{E}} \mathrm{h}^{-1}|\llbracket \eta \eta|^{2} \mathrm{~d} s\right)^{\frac{1}{2}} \\
& \leq C\left(\sum_{K \in \mathcal{T}} h_{K}\|\nabla v\|_{L^{2}(\partial K)}^{2}\right)^{\frac{1}{2}}\|\eta\|_{\mathrm{DG}} .
\end{aligned}
$$

Applying a suitable trace estimate for polynomials, we obtain

$$
\left|T_{3}\right| \leq C\left(\sum_{K \in \mathcal{T}}\|\nabla v\|_{L^{2}(K)}^{2}\right)^{\frac{1}{2}}\|\eta\|_{\mathrm{DG}} \leq C\|\eta\|_{\mathrm{DG}}\|v\|_{\mathrm{DG}} .
$$


Finally, we estimate $T_{4}$ :

$$
\left|T_{4}\right| \leq\left(\int_{\mathcal{E}} \mathrm{h}^{-1}|\llbracket \eta \eta \rrbracket|^{2} \mathrm{~d} s\right)^{\frac{1}{2}}\left(\int_{\mathcal{E}} \mathrm{h}^{-1}|\llbracket \xi \xi \rrbracket|^{2} \mathrm{~d} s\right)^{\frac{1}{2}} \leq C\|\eta\|_{\text {DG }}\|v\|_{\mathrm{DG}} .
$$

Combining (18)-(22), yields the desired estimate.

We are now prepared to prove the main result of this paper.

Theorem 5.2 Let $p \in(1,2]$ and $\gamma>0$ be sufficiently large to ensure that $a_{\mathrm{DG}}$ is coercive on $V_{\mathrm{DG}}(\mathcal{T})$. Furthermore, suppose that the exact solution $u$ of (1)-(2) belongs to $W^{l+1, p}(\Omega, \mathcal{T})$, where $l \geq 1$ is the polynomial degree in the $D G$ space $V_{\mathrm{DG}}(\mathcal{T})$. Then, the error $e_{\mathrm{DG}}$ from (17) between the exact solution and the $D G$ solution $u_{\mathrm{DG}} \in V_{\mathrm{DG}}(\mathcal{T})$ from (7) satisfies the a priori error bound

$$
\left\|u-u_{\mathrm{DG}}\right\|_{\mathrm{DG}} \leq C\left(\sum_{K \in \mathcal{T}} h_{K}^{p(l+1)-2}\|u\|_{W^{l+1, p}(K)}^{p}\right)^{\frac{1}{p}} .
$$

Here, $C>0$ is a constant independent of $\boldsymbol{h}$.

Proof Recalling the decomposition (17), and using the triangle inequality, implies

$$
\left\|e_{\mathrm{DG}}\right\|_{\mathrm{DG}} \leq\|\eta\|_{\mathrm{DG}}+\|\xi\|_{\mathrm{DG}} .
$$

We will bound $\|\mid \eta\|_{\text {DG }}$ first. There holds

$$
\begin{aligned}
\|\eta\|_{\mathrm{DG}}^{2} & =\left\|\nabla_{h} \eta\right\|_{L^{2}(\Omega)}^{2}+\gamma \int_{\mathcal{E}} \mathrm{h}^{-1}|\llbracket \eta \rrbracket|_{L^{2}(e)}^{2} \\
& \leq C \sum_{K \in \mathcal{T}}\left(\|\nabla \eta\|_{L^{2}(K)}^{2}+h_{K}^{-1}\|\eta\|_{L^{2}(\partial K)}^{2}\right) .
\end{aligned}
$$

By the trace inequality and elementwise scaling, we obtain

$$
\|\eta\|_{L^{2}(\partial K)}^{2} \leq C\left(h_{K}^{-1}\|\eta\|_{L^{2}(K)}^{2}+h_{K}\|\nabla \eta\|_{L^{2}(K)}^{2}\right) \quad \forall K \in \mathcal{T},
$$

and thus,

$$
\|\eta\|_{\mathrm{DG}}^{2} \leq C \sum_{K \in \mathcal{T}}\left(h_{K}^{-2}\|\eta\|_{L^{2}(K)}^{2}+\|\nabla \eta\|_{L^{2}(K)}^{2}\right) .
$$

We will now estimate $\|\xi\|_{\mathrm{DG}}$. Notice that $a_{\mathrm{DG}}$ is coercive on $V_{\mathrm{DG}}(\mathcal{T})$ if $\gamma$ is sufficiently large. Hence, since $\xi \in V_{\mathrm{DG}}(\mathcal{T})$, and using (12), there holds

$$
C\|\xi\|_{\mathrm{DG}}^{2} \leq a_{\mathrm{DG}}(\xi, \xi)=a_{\mathrm{DG}}\left(e_{\mathrm{DG}}-\eta, \xi\right)=-a_{\mathrm{DG}}(\eta, \xi) .
$$

Moreover, using Proposition 5.1, leads to

$$
\begin{aligned}
\|\xi\|_{\mathrm{DG}}^{2} & \leq C\left|a_{\mathrm{DG}}(\eta, \xi)\right| \\
& \leq C\left(\|\eta\|_{\mathrm{DG}}+\left(\sum_{K \in \mathcal{T}} h_{K}^{p-2}\left(\|\nabla \eta\|_{L^{p}(K)}+h_{K}\left\|\mathrm{D}^{2} \eta\right\|_{L^{p}(K)}\right)^{p}\right)^{\frac{1}{p}}\right)\|\xi\|_{\mathrm{DG}} .
\end{aligned}
$$


Dividing both sides of the above inequality by $\|\xi\|_{\text {DG }}$, we find that

$$
\|\xi\|_{\mathrm{DG}} \leq C\left(\|\eta\|_{\mathrm{DG}}+\left(\sum_{K \in \mathcal{T}} h_{K}^{p-2}\left(\|\nabla \eta\|_{L^{p}(K)}+h_{K}\left\|\mathrm{D}^{2} \eta\right\|_{L^{p}(K)}\right)^{p}\right)^{\frac{1}{p}}\right) .
$$

Therefore, combining (24) and (26), and applying (25), yields

$$
\begin{aligned}
\left\|e_{\mathrm{DG}}\right\|_{\mathrm{DG}} \leq & C\left(\|\eta\|_{\mathrm{DG}}+\left(\sum_{K \in \mathcal{T}} h_{K}^{p-2}\left(\|\nabla \eta\|_{L^{p}(K)}+h_{K}\left\|\mathrm{D}^{2} \eta\right\|_{L^{p}(K)}\right)^{p}\right)^{\frac{1}{p}}\right) \\
\leq & C\left(\left(\sum_{K \in \mathcal{T}}\left(h_{K}^{-2}\|\eta\|_{L^{2}(K)}^{2}+\|\nabla \eta\|_{L^{2}(K)}^{2}\right)\right)^{\frac{1}{2}}\right. \\
& \left.+\left(\sum_{K \in \mathcal{T}} h_{K}^{p-2}\left(\|\nabla \eta\|_{L^{p}(K)}+h_{K}\left\|\mathrm{D}^{2} \eta\right\|_{L^{p}(K)}\right)^{p}\right)^{\frac{1}{p}}\right) .
\end{aligned}
$$

Now, let $P=\Pi$ in (17), where $\Pi$ is the interpolant from Proposition 4.1. Using (15) and (16), we finally obtain

$$
\left\|e_{\mathrm{DG}}\right\|_{\mathrm{DG}} \leq C\left(\sum_{K \in \mathcal{T}} h_{K}^{p(l+1)-2}\|u\|_{W^{l+1, p}(K)}^{p}\right)^{\frac{1}{p}}
$$

This shows (23).

Remark 5.3 Provided that the exact solution of (1)-(2) is in $W^{l+1, p}(\Omega)$, the error estimate (23) reads

$$
\left\|u-u_{\mathrm{DG}}\right\|_{\mathrm{DG}} \leq C\left(\max _{K \in \mathcal{T}} h_{K}\right)^{l+1-\frac{2}{p}}\|u\|_{W^{l+1, p}(\Omega)} .
$$

This estimate is optimal for the given norms.

We complete this section by presenting the following result.

Corollary 5.4 Suppose that $f \in L^{p}(\Omega)$ with $p>1$ sufficiently small (cf. Proposition 2.3). Additionally, let $\gamma$ be large enough so that the DGFEM (7) has a unique solution. Furthermore, let $\mathcal{T}$ be quasi-uniform of mesh size $h:=\max _{K \in \mathcal{T}} h_{K}>0$. Then, the $D G$ method (7) based on piecewise linear polynomial functions for the approximation of (1)(2) converges at a positive algebraic rate with respect to the number of degrees of free$\operatorname{dom} N=\operatorname{dim} V_{\mathrm{DG}}(\mathcal{T})$. More precisely, there holds

$$
\left\|u-u_{\mathrm{DG}}\right\|_{\mathrm{DG}} \leq C N^{-1+\frac{1}{p}}\|f\|_{L^{p}(\Omega)},
$$

where $C>0$ is a constant independent of $N$ and $u$.

Proof This follows directly from Remark 5.3, from the fact that $N \sim h^{-2}$, and from Proposition 2.3 . 
Table 1 Convergence rates for NIPG with piecewise linears, quadratics and cubics

\begin{tabular}{lllll}
\hline$k$ & $\alpha=2^{-k}$ & rate for $l=1$ & rate for $l=2$ & rate for $l=3$ \\
\hline 0 & 1.00 & $9.18 \times 10^{-1}$ & $9.96 \times 10^{-1}$ & $9.96 \times 10^{-1}$ \\
1 & $5.00 \times 10^{-1}$ & $4.94 \times 10^{-1}$ & $4.97 \times 10^{-1}$ & $4.97 \times 10^{-1}$ \\
2 & $2.50 \times 10^{-1}$ & $2.47 \times 10^{-1}$ & $2.47 \times 10^{-1}$ & $2.47 \times 10^{-1}$ \\
3 & $1.25 \times 10^{-1}$ & $1.22 \times 10^{-1}$ & $1.22 \times 10^{-1}$ & $1.22 \times 10^{-1}$ \\
4 & $6.25 \times 10^{-2}$ & $6.02 \times 10^{-2}$ & $6.05 \times 10^{-2}$ & $6.03 \times 10^{-2}$ \\
\hline
\end{tabular}

\section{Numerical Experiments}

We will now illustrate our theoretical results with a numerical example. To this end, let $\Omega=$ $(0,1 / 4)^{2}$. Furthermore, choose $f$ in (1) such that

$$
u(x, y)=x(x-0.25) y(y-0.25) r^{-2+\alpha}
$$

becomes the exact solution of (1)-(2). Here, $\alpha \in(0,1]$ is a constant, and $r=\left(x^{2}+y^{2}\right)^{\frac{1}{2}}$ denotes the distance to the origin. We note that

$$
u \in W_{0}^{1,2}(\Omega) \cap W^{2, p}(\Omega),
$$

for all $p \in\left(1, \frac{2}{2-\alpha}\right) \subseteq(1,2)$. Thus, for the DG method with linear polynomials, $l=1$, on quasi-uniform meshes of mesh width $h$, we expect (cf. Remark 5.3) that

$$
\left\|u-u_{\mathrm{DG}}\right\|_{\mathrm{DG}} \sim h^{2-\frac{2}{p}} \sim h^{\alpha} .
$$

The domain is initially partitioned into triangles with $h=1 / 8$. Successive uniform refinements are performed to numerically determine the convergence rate. Table 1 shows the rates obtained with the finest meshes for various values of $\alpha$. The non-symmetric interior penalty Galerkin method (NIPG) is used with $\theta=-1$ and $\gamma=1$. The polynomial degree $l$ takes the values one, two and three.

We observe, as predicted by the theory, that the rate is lost as $\alpha$ tends to 0 . The rate is independent of the polynomial degree, which is not surprising since $u \in W^{2, p}(\Omega)$ only. We note, however, that the convergence rates are attained on coarser meshes for higher polynomial degrees.

Table 2 presents the numerical rates obtained with the symmetric interior penalty Galerkin (SIPG) method $(\theta=1)$. The penalty parameter is chosen to guarantee coercivity of the method, cf., e.g., [14]: $\gamma=6$ for $l=1, \gamma=18$ for $l=2$, and $\gamma=36$ for $l=3$. We obtain similar results as for the NIPG method. For the case $\alpha=2^{-4}$, the obtained rates on the finest meshes have not converged yet to the theoretical value. The size of the linear systems produced on further refined meshes exceeds our solver capabilities.

For comparison, we solve the same problem with the continuous finite element method of order one, and obtain similar results (see Table 3 ). We also note that the numerical rate for the $L^{2}$ error is $\mathcal{O}\left(h^{1+\alpha}\right)$ for all methods and all degrees considered above.

\section{Conclusions}

In this paper we have studied the convergence of DG approximations of the Poisson equation with low regularity solutions. We have obtained optimal a priori error estimates (in the standard DG norm) in terms of $L^{p}$-based norms of the solution. The results are confirmed with a 
Table 2 Convergence rates for SIPG with piecewise linears, quadratics and cubics

\begin{tabular}{lllll}
\hline$k$ & $\alpha=2^{-k}$ & rate for $l=1$ & rate for $l=2$ & rate for $l=3$ \\
\hline 0 & 1.00 & $9.05 \times 10^{-1}$ & $9.93 \times 10^{-1}$ & $9.79 \times 10^{-1}$ \\
1 & $5.00 \times 10^{-1}$ & $4.91 \times 10^{-1}$ & $4.95 \times 10^{-1}$ & $4.94 \times 10^{-1}$ \\
2 & $2.50 \times 10^{-1}$ & $2.45 \times 10^{-1}$ & $2.45 \times 10^{-1}$ & $2.45 \times 10^{-1}$ \\
3 & $1.25 \times 10^{-1}$ & $1.21 \times 10^{-1}$ & $1.20 \times 10^{-1}$ & $1.21 \times 10^{-1}$ \\
4 & $6.25 \times 10^{-2}$ & $5.87 \times 10^{-2}$ & $5.79 \times 10^{-2}$ & $5.91 \times 10^{-2}$ \\
\hline
\end{tabular}

Table 3 Convergence rates for the finite element method with continuous piecewise linears

\begin{tabular}{lll}
\hline$k$ & $\alpha=2^{-k}$ & rate \\
\hline 0 & 1.00 & $9.24 \times 10^{-1}$ \\
1 & $5.00 \times 10^{-1}$ & $5.00 \times 10^{-1}$ \\
2 & $2.50 \times 10^{-1}$ & $2.49 \times 10^{-1}$ \\
3 & $1.25 \times 10^{-1}$ & $1.24 \times 10^{-1}$ \\
4 & $6.25 \times 10^{-2}$ & $6.18 \times 10^{-2}$ \\
\hline
\end{tabular}

numerical example. We expect that the techniques used in this paper may be extended to the error analysis of discontinuous Galerkin discretizations of more general linear and nonlinear second-order elliptic PDE. In particular, a number of nonlinear elliptic problems (e.g., the $p$-Laplace equation) feature solutions in $W^{1, p}$, with $p<2$.

\section{References}

1. Adams, R.A., Fournier, J.J.F.: Sobolev Spaces. Elsevier, Amsterdam (2003)

2. Arnold, D.N.: An interior penalty finite element method with discontinuous elements. SIAM J. Numer. Anal. 19, 742-760 (1982)

3. Arnold, D.N., Brezzi, F., Cockburn, B., Marini, L.D.: Unified analysis of discontinuous Galerkin methods for elliptic problems. SIAM J. Numer. Anal. 39, 1749-1779 (2001)

4. Baumann, C.E., Oden, J.T.: A discontinuous $h p$-finite element method for convection-diffusion problems. Comput. Methods Appl. Mech. Eng. 175, 311-341 (1999)

5. Brenner, S.C., Scott, L.R.: The Mathematical Theory of Finite Element Methods, 3rd edn. Texts in Applied Mathematics, vol. 15. Springer, New York (2008)

6. Castillo, P., Cockburn, B., Perugia, I., Schötzau, D.: An a priori error analysis of the local discontinuous Galerkin method for elliptic problems. SIAM J. Numer. Anal. 38, 1676-1706 (2000)

7. Ciarlet, P.G.: The Finite Element Method for Elliptic Problems. Classics in Applied Mathematics, vol. 40. SIAM, Philadelphia (2002)

8. Dauge, M.: Elliptic Boundary Value Problems on Corner Domains. Lecture Notes in Math., vol. 1341. Springer, Berlin (1988)

9. Dawson, C., Sun, S., Wheeler, M.F.: Compatible algorithms for coupled flow and transport. Comput. Methods Appl. Mech. Eng. 193(23-26), 2565-2580 (2004)

10. Di Pietro, D.A., Ern, A.: Discrete functional analysis tools for discontinuous Galerkin methods with application to the incompressible Navier-Stokes equations. Math. Comput. 79, 1303-1330 (2010)

11. Hardy, G.H., Littlewood, J.E., Pólya, G.: Inequalities, 2nd edn. Cambridge University Press, Cambridge (1988). Reprint of the 1952 edition

12. Houston, P., Schwab, C., Süli, E.: Discontinuous $h p$-finite element methods for advection-diffusionreaction problems. SIAM J. Numer. Anal. 39, 2133-2163 (2002)

13. Quarteroni, A., Valli, A.: Numerical Approximation of Partial Differential Equations. Springer Series in Computational Mathematics, vol. 23. Springer, Berlin (2008)

14. Rivière, B.: Discontinuous Galerkin Methods for Solving Elliptic and Parabolic Equations, Theory and Implementation. Frontiers in Applied Mathematics. SIAM, Philadelphia (2008) 
15. Rivière, B., Wheeler, M.F., Girault, V.: A priori error estimates for finite element methods based on discontinuous approximation spaces for elliptic problems. SIAM J. Numer. Anal. 39(3), 902-931 (2001) (electronic)

16. Schötzau, D., Wihler, T.P.: Exponential convergence of mixed $h p$-DGFEM for Stokes flow in polygons. Numer. Math. 96, 339-361 (2003)

17. Scott, L.R., Zhang, S.: Finite element interpolation of nonsmooth functions satisfying boundary conditions. Math. Comput. 54(190), 483-493 (1990)

18. Wheeler, M.F.: An elliptic collocation finite element method with interior penalties. SIAM J. Numer. Anal. 15, 152-161 (1978)

19. Wihler, T.P.: Discontinuous Galerkin FEM for elliptic problems in polygonal domains. PhD thesis, Swiss Federal Institute of Technology Zurich (2002). Diss. ETH No. 14973

20. Wihler, T.P.: Locking-free DGFEM for elasticity problems in polygons. IMA J. Numer. Anal. 24(1), 45-75 (2004)

21. Wihler, T.P., Frauenfelder, P., Schwab, C.: Exponential convergence of the $h p$-DGFEM for diffusion problems. Comput. Math. Appl. 46, 183-205 (2003) 